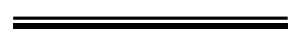

\title{
Constructing Compact and Effective Graphs for Recommender Systems via Node and Edge Aggregations
}

\author{
Sangkeun Lee ${ }^{\mathrm{a}}$, Minsuk Kahng ${ }^{\mathrm{b}}$, Sang-goo Lee ${ }^{\mathrm{c}}$ \\ ${ }^{a}$ Computational Science and Engineering Division, Oak Ridge National Laboratory \\ ${ }^{b}$ College of Computing, Georgia Institute of Technology \\ ${ }^{c}$ Department of Computer Science and Engineering, Seoul National University
}

\begin{abstract}
Exploiting graphs for recommender systems has great potential to flexibly incorporate heterogeneous information for producing better recommendation results. As our baseline approach, we first introduce a naïve graph-based recommendation method, which operates with a heterogeneous log-metadata graph constructed from user log and content metadata databases. Although the naïve graph-based recommendation method is simple, it allows us to take advantages of heterogeneous information and shows promising flexibility and recommendation accuracy. However, it often leads to extensive processing time due to the sheer size of the graphs constructed from entire user log and content metadata databases. In this paper, we propose node and edge aggregation approaches to constructing compact and effective graphs called 'Factor-Item bipartite graphs' by aggregating nodes and edges of a log-metadata graph. Experimental results using real world datasets indicate that our approach can significantly reduce the size of graphs exploited for recommender systems without sacrificing the recommendation quality.
\end{abstract}

Keywords: graph, heterogeneity, recommendation, aggregation, random-walk, ranking

\section{Introduction}

Recommender systems aim to help users find unobvious, but preferable information hidden in the long-tail. There have been various research efforts regarding recommender systems by both industry and academia. So far, finding out an effective utility function $u:$ User $\times$ Item $\rightarrow$ Utility which estimates users' preferences on unseen items using based on their prior ratings is the most common problem formulation in the research domain of recommender systems. Thanks to its simplicity and clear formalized definition, it motivated many scientists and practitioners to produce a wide range of outcomes including algorithms, systems, tools and so on; however, it has limitation because it is not trivial to incorporate heterogeneous information such as contextual information, metadata of items or users, and relationships among entities.

To resolve such limitation, several context-aware recommender systems have been proposed (Panniello et al., 2014; Baltrunas and Ricci, 2014; Codina et al., 2013; Adomavicius et al., 2011; Kahng et al., 2011a; Lee et al., 2010; Adomavicius et al., 2005). On the other hand, several recommender systems which exploit graphs as their underlying data structure also have been proposed (Demovic et al., 2013; Lee et al., 2013; Xiang et al., 2010; Cheng et al., 2007; Gori and Pucci, 2007; Fouss et al., 2007; Huang et al., 2002). Majority of these existing graph-based approaches mainly focused on improving recommendation quality, but more important advantage of exploiting graph structures

URL: lees4@ornl.gov (Sangkeun Lee), kahng@gatech.edu (Minsuk Kahng), sglee@snu.ac.kr (Sang-goo Lee) 
for recommender systems is that we can achieve the flexibility of incorporating heterogeneous information into the recommendation process.

In this paper, as our baseline, we introduce a naïve graph-based recommendation method which exploits a logmetadata graph as its underlying data structure. A log-metadata graph is a heterogeneous graph which is a type of graph composed of multiple types of nodes and edges. To construct a log-metadata graph, the naïve method transforms tuples of user $\log$ and content metadata tables into nodes and foreign key relationship between tuples into edges, respectively (Aditya et al., 2002). A log table implicitly implies users preferences on items, since a users repeated item consumptions on the same or similar items are valuable information for recommendation. In addition, metadata table of items includes information that can be used to identify the similarity among the items. Note that log and metadata tables are prevalent in many application databases, and there are a number of examples where we can apply the approach (e.g., hotel recommendation based on previous booking history, news recommendation based on news click logs, menu recommendation for a customer using his previous order history, etc.). Graph structure provides great flexibility so that any new type of nodes or edges can be incorporated without modifying the database schema unlike the relational model. Then, recommendation results are generated by ranking nodes in the constructed log-metadata graph, exploiting Personalized PageRank (Haveliwala, 2003) for a given recommendation query node (e.g., an active user node).

Although the naïve method is simple and it is mainly based on existing approaches, it enables us to flexibly incorporate heterogeneous information into the recommendation procedure and produce promising recommendation results. However, we noticed that the size of log-metadata graph is often very large because the graph is constructed from entire user log and content metadata databases. Processing such large graphs requires extensive computing power and processing time. To resolve this problem, we present a recommendation method based on Factor-Item bipartite graphs constructed by aggregating nodes and edges of a log-metadata graph.

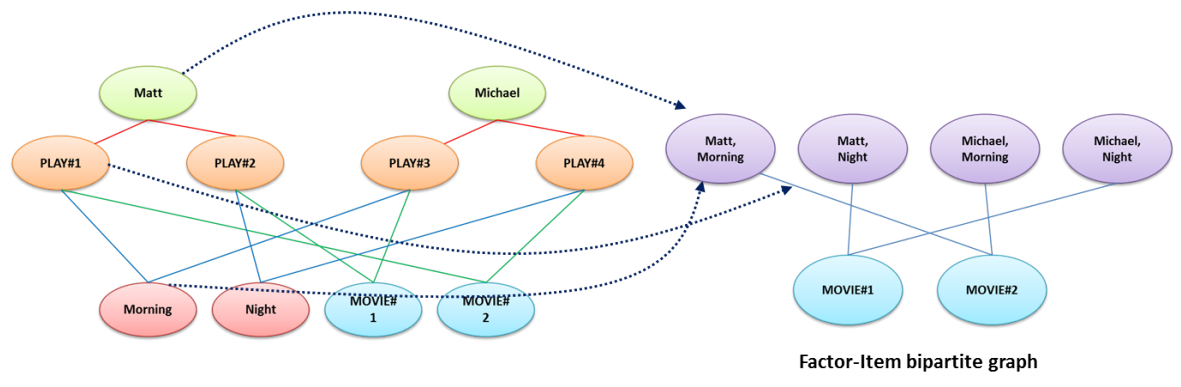

Figure 1. Constructing a Factor-Item bipartite graph by Node and Edge Aggregations

Figure 1 shows an example of constructing a Factor-Item bipartite graph from a log-metadata graph. Performing node and edge aggregation on a log-metadata graph will generate a reduced, smaller-sized graph so that we can perform recommendation more efficiently. Recommendation service providers can define factors that decide how to aggregate nodes and edges of a log-metadata graph. The naïve method's recommendation results generated by the Personalized PageRank (Haveliwala, 2003) are deterministic for given queries, meaning that the method will not reflect service providers' different intentions depending on their needs or situations. Like the naïve recommendation method, we use Personalized PageRank (Haveliwala, 2003) to define a utility function for scoring and ranking nodes; however, depending on different factors, we can construct various Factor-Item bipartite graphs generating recommendations with different semantics (e.g., collaborative filtering, content-based filtering, and context-aware collaborative filtering).

To validate our proposed methods, we compare our two proposed graph-based recommendation methods with the other existing recommendation methods using real datasets. The experimental results show that our proposed methods show better or comparable recommendation quality compared to existing recommendation methods. By constructing Factor-Item bipartite graphs, we could significantly reduce the size of exploited graph size compared to the naïve method without sacrificing the recommendation results. In the best case, it even generates better recommendation performance in terms of accuracy. 


\section{Related Work}

Most traditional recommender systems (e.g., Collaborative Filtering (CF) (Herlocker et al., 1999; Koren, 2008; Cremonesi et al., 2010), or Content-Based Filtering (CBF) (Pazzani and Billsus, 2007; Whitman and Lawrence, 2002) operate in two-dimensional space User $\times$ Item. However, in many real world applications, it is not sufficient to assume this limited world model that consists of only two types of entities User and Item. There have been a number of studies on incorporating heterogeneous information into recommender systems.

Since Adomavicius et al. (2005) introduced the concept of multidimensional recommendation by generalizing the recommendation problem to the problem of dealing with multidimensional space $D_{1} \times \ldots \times D_{n}$, several multidimensional recommendation approaches have been proposed. Adomavicius et al. proposed a reduction-based $\mathrm{CF}$ (Adomavicius et al., 2005). It first filters out ratings that do not match the current context. Then, conventional two-dimensional CF algorithms are performed on the reduced space. Disjunction-based CF (Lee et al., 2010) was proposed to tackle the sparsity problem which can be caused by the reduction-based CF. In this approach, instead of using all context dimensions at the same time, the algorithm disjunctively uses each contextual dimension and merges the results. Kahng et al. (2011a) proposed a similar approach by taking a Learning-to-Rank approach to compute the weights of contextual effects on recommendation. By getting hints from the matrix factorization based CF approaches, several tensor factorization techniques for multidimensional cases have been introduced (Rendle, 2010; Karatzoglou et al., 2010); however, the complexity of the algorithm is exponential to the number of dimensions, which makes the algorithm infeasible in many real world cases.

Apart from the multidimensional approaches, several graph-based recommender systems have been introduced. In detail, Fouss et al. (2007) modeled the recommendation problem as measuring dissimilarities between nodes of a people-movie bipartite graph. The authors compared several random walk based quantities (e.g. L+ (Schölkopf and Smola, 2002), average commute time (Göbel and Jagers, 1974)) for recommendation. Gori and Pucci (2007) presented a graph-based method named ItemRank which is a random-walk based scoring algorithm. It operates with a movie network linked by edges whose weights are assigned to be the number of users who watch both movies which the edge connects.

Later, researchers presented several approaches to exploting graphs for dealing with multidimensional recommendation or combining different recommendation techniques. Xiang et al. (2010) presented a graph-based recommendation which aims to improve recommendation accuracy by mixing users' long-term and short-term preferences. Lee et al. (2011) presented how to construct and use a bipartite graph for more general multidimensional recommendation. Demovic et al. (2013) showed graph traversal algorithms designed for movie recommendation using movie graphs.

Flexibility is the capability of enabling easy customization of incorporating heterogeneous information and generate various recommendation results with different semantics, and it is one of very important criteria of recommender systems (Koutrika et al., 2009). However, majority of multidimensional and graph-based recommendation approaches including recent researches only focus on increasing the measurable quality (e.g., accuracy, diversity, etc.) of recommender systems, and they lack of considerations for achieving flexibility of recommender systems (Fouss et al., 2007; Gori and Pucci, 2007; Xiang et al., 2010; Demovic et al., 2013; Baltrunas and Ricci, 2014; Panniello et al., 2014).

In this paper, we present how to achieve flexibility by customizing graph construction and node ranking. It should be noted that, in many cases, graphs constructed for recommender systems are very large. Thus, we should be able to process large-scale graphs to practically employ graph-based recommendation approaches. So far, there have been only system-oriented approaches to tackle the problem such as Pregel (Malewicz et al., 2010) and GraphX (Xin et al., 2013) that aim to scale up the node ranking process by taking advantages of distributed parallel computing. We look at the problem of dealing with large-scale data sets with a new perspective. Instead of trying to scale up the graph processing, we focus on reducing the problem size itself by constructing compact graphs for recommendation without losing much recommendation accuracy. Note that there have been few existing graph-based recommender systems to enable flexibility of recommender systems exploiting paths in graphs (Lee et al., 2013; Kahng et al., 2011b; Yao et al., 2014); however, they did not address the problem of constructing compact graphs for recommendation.

As an extended work of our previously published conference paper (Lee et al., 2011), we introduce the concepts of a naïve graph-based recommendation method using the log-metadata graph, node and edge aggregation operations for constructing compact and effect graphs, called Factor-Item bipartite graphs. We also included additional experimental results for thorough comparisons of our proposed methods with the other existing recommendation methods. 


\section{Preliminaries}

A heterogeneous graph is a type of graph which is composed of multiple types of nodes and edges (Sun and Han, 2012). Generally, nodes represent entities, and edges represent relationships. There are many variations of heterogeneous graphs depending on whether nodes or edges have weights or properties or whether edges have directions. The heterogeneous graph we exploit in this paper is formally defined as follows.

Definition 1. Heterogeneous Graph. A heterogeneous graph is a directed graph $G=\left(V, E, T_{V}, T_{E}\right)$, where $V$ is a finite set of nodes, $E \subseteq V \times V$ is a finite multi-set of edges, $T_{V}$ is a finite set of node types, and $T_{E}$ is a finite set of edge types. Each node is mapped to a node type by a node type mapping function $\phi_{V}: V \rightarrow T_{V}$, and each edge is mapped to an edge type by an edge type mapping function $\phi_{E}: E \rightarrow T_{E}$. Each node $v_{i} \in V$ can have a set of (attribute, value) pairs which describe the properties of the node. Each edge $e_{k}\left(v_{i}, v_{j}\right) \in E$ has a corresponding weight value $w_{k}$.

In order to describe the schema-level information of a heterogeneous graph, we define the graph schema of a heterogeneous graph as follows.

Definition 2. Graph Schema The graph schema of a heterogeneous graph $G=\left(V, E, T_{V}, T_{E}\right)$ is a schema-level template for the heterogeneous graph, and it is defined as a directed graph $G_{S}=\left(T_{V}, T_{E}\right)$, where $T_{V}$ is a finite set of node types, and $T_{E} \subseteq T_{V} \times T_{V}$ is a finite set of edge types.

We can create a heterogeneous graph based on user log and metadata databases of a recommendation domain. For example, in the domain of music recommendation, user log databases are a set of tables which include users' music listening histories, and metadata databases are a set of tables describing features of albums, songs, artists, and so on. We named such heterogeneous graph log-metadata graph. Then, we need to formulate the recommendation problem as the problem of ranking recommendation target type nodes (e.g., Song nodes) on a log-metadata graph for a given recommendation needs query (e.g., a User node), where the query is described as a set of query nodes. We formally formulate the problem as follows.

Definition 3. Graph-based Top-k Recommendation. For a given log-metadata graph $G=\left(V, E, T_{V}, T_{E}\right)$, a recommendation needs query is given as $Q=(N, \tau, k)$, where $N=\left\{q_{1}, \ldots, q_{|N|}\right\} \subseteq V$ is a set of query nodes, $\tau \in T_{V}$ is a target type, and $k$ is the number of recommended items for the query. The query node set $N$ describes a user's needs for recommendation, and the target type $\tau$ describes a type of nodes that are recommendation candidates. Let $\Psi$ be the set of all possible query node sets $N$ and $V_{\tau}$ be the set of all target nodes $v \in V$ where $\phi_{V}(v)=\tau$. Then, we let $u$ : $\Psi$ $\times V_{\tau} \rightarrow \mathfrak{R}$ be a utility function that measures the usefulness of candidate nodes $v \in V_{\tau}$ to the given query set $N \in Q$, where $\mathfrak{R}$ is totally ordered set. For a given recommendation query $Q=(N, \tau, k)$, nodes $v \in V_{\tau}$ that have top- $k$ highest utility scores $u(Q, v)$ are selected as recommendation results.

\section{A Naïve Method for Graph-based Recommendation}

In this section, we introduce a naïve method as a straightforward approach to graph-based recommendation. The method exploits the existing techniques to build a log-metadata graph (Aditya et al., 2002) and rank nodes on the constructed graph (Haveliwala, 2003). The naïve method can incorporate rich information into the recommendation procedure, but it still has several limitations to be practically exploited. We discuss limitations at the end of this section.

\subsection{Constructing a log-metadata Graph}

For the naïve graph-based recommender system,, we need to construct a log-metadata describing the recommendation application domain database (e.g., log and metadata tables). We assume that the datasets are stored in a relational database. The schema of the relational database can be mapped to an ER (entity-relationship) diagram which is a graph. Thus, we can transform the relational tables into a heterogeneous graph using the following heuristic rule. We assume that each tuple represents an entity, so every tuple in the database is transformed into a node, and attribute value pairs of a tuple can be transformed into attribute-value pairs of the corresponding node. Each link from foreign key to primary key is transformed into a directed edge between the corresponding nodes (Aditya et al., 2002). We assign the same weight value ' 1 ' to every node. Figure 2 shows an example of constructing a log-metadata graph from relational tables. 


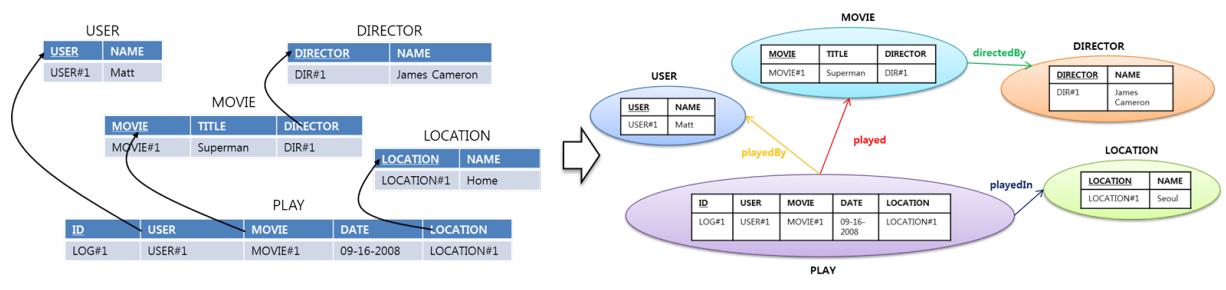

Figure 2. Constructing a log-metadata graph from relational tables

\subsection{Defining a Utility Function}

To perform graph-based top-k recommendation, we need to define a utility function which quantifies the utilities of nodes for a given query. We can adopt existing node ranking measures to define the utility function. There are many node ranking measures defined on graphs from simple ones such as the shortest path (Floyd, 1962) and maximum flow (Goldberg and Tarjan, 1988) to more sophisticated measures based on the random-walk model (Page et al., 1999; Balmin et al., 2004; Haveliwala, 2003; Jeh and Widom, 2002; Shi et al., 2012). Among the measures, we adopt Personalized PageRank (Haveliwala, 2003) to define the utility function. Personalized PageRank (Haveliwala, 2003) reflects a wide range of properties of a graph such as distance between query nodes and target nodes, number of paths between query nodes and target nodes, node authorities, and so on. Thus, Personalized PageRank can identify the nodes that are closely related to the query nodes and important entities like popular items, items tagged by many users, heavy users, and so on. In addition, Personalized PageRank can be exploited to measure the relevance between multiple nodes and a target node, although many existing node ranking measures are defined only between 1-to-1 relationships (Jeh and Widom, 2002; Shi et al., 2012; Sun et al., 2011; Floyd, 1962).

By ignoring the node types, edge types and values of node attributes, a log-metadata graph $G=\left(V, E, T_{V}, T_{E}\right)$ can be characterized by a $|V| \times|V|$ adjacency matrix $M$, where it is defined as follows:

$$
M(i, j)= \begin{cases}\forall e_{k} \Sigma w_{k} & , \text { if } \exists e_{k}\left(v_{i}, v_{j}\right) \in E \\ 0 & , \text { otherwise }\end{cases}
$$

where $e_{k}\left(v_{i}, v_{j}\right)$ is an edge connecting $v_{i}$ and $v_{j} \in V$, ignoring the edge direction, and $M(i, j)$ is the $M$ 's element of the $i$-th row and the $j$-th column.

Random walks on the constructed graph can be considered as a Markov-chain, and it can be characterized by a transition matrix $P$ derived from the matrix $M$ as follows:

$$
P(i, j)= \begin{cases}\frac{M(i, j)}{\sum_{v_{k} \in \operatorname{coutink}\left[v_{i}\right]} M(i, k)} & , \text { if outlink }\left[v_{i}\right] \neq \phi \\ 0 & , \text { otherwise }\end{cases}
$$

where $P(i, j)$ is the $P$ 's element of the $i$-th row and the $j$-th column, and $M(i, j)$ is the $M$ 's element of the $i$-th row and the $j$-th column.

Personalized PageRank is achieved by the equation $\vec{r}=c P^{T} \vec{r}+(1-c) \vec{t}$, where i-th element of $\vec{r}$ is the Personalized PageRank score for node $v_{i}, \vec{t}$ is a teleport vector, and $c$ is a damping factor constant that is normally given as 0.85 . Intuitively, Personalized PageRank score of a node is the probability of random walkers being at the node after enough number of random walk with restart iterations. At each iteration, the random walker has two options that are transition or restart. With probability $c$, the random walker follows an adjacent edge, otherwise the random walker jumps to a node $v_{i}$ with probability $t_{i}$, where $t_{i}$ is i-th element of $\vec{t}$.

A query $Q$ for a graph-based recommendation is given as $Q=(N, \tau, k)$. For the given query $Q=\left(N=\left\{v_{1}, v_{2}, \ldots, v_{|N|}\right\}\right.$, $\tau, k$ ) we define a vector $\breve{\vec{q}}$ as follows:

$$
\breve{q}_{i}= \begin{cases}1 & , \text { if } v_{i} \in N \\ 0 & , \text { otherwise }\end{cases}
$$


Then, we normalize the vector $\breve{\vec{q}}$ making the values to sum up to 1 , and we use the normalized vector $\vec{q}$ as the teleport vector in the Personalized PageRank equation. So, the ranking score vector is calculated as $\vec{r}=c P^{T} \vec{r}+(1-c) \vec{q}$. Consequently, we can use the rank $r_{i}$ for each node $v_{i}$ whose $\phi_{V}\left(v_{i}\right)=\tau$ so that we can generate top-k recommendation results for the query.

By using the naïve method, we can incorporate rich and heterogeneous information into the recommendation procedure, and various recommendations with different types of inputs and outputs can be coherently provided using the same log-metadata graph. However, the naïve method still has several limitations. First, a log-metadata graph straightforwardly transformed and constructed from entire user log and metadata tables in a relational database is often very large. All tuples in the tables are transformed into nodes. As the nodes transformed from a tuple usually have n-nary relationships with the other nodes, the number of edges in the graph also becomes very large. Consequently, it may lead to inefficiency in terms of processing time. Secondly, recommendation results generated by the naïve method are deterministic for given queries, although recommendation service providers may want to adjust factors that affect recommendation results or generate recommendation results with different semantics. For example, service provider may want to generate a list of recommended songs that have similar tags with the songs that a user often listened, or a list of songs that are preferred by the other similar users. To resolve such limitations, in the following section, we propose our node and edge aggregation approach to constructing compact and effective graphs with different intentions of recommendation service providers.

\section{Constructing Compact Graphs for Recommender Systems}

In this section, we propose an efficient and effective graph structure for graph-based recommender systems, named Factor-Item bipartite graph that is a bipartite graph like the graphs exploited in several existing graph-based recommender systems (Fouss et al., 2007; Davidson et al., 2010). However, unlike the bipartite graphs used in the existing systems, rich and heterogeneous information (e.g., time, location, contents metadata, etc.) can be incorporated into Factor-Item bipartite graphs. A Factor-Item bipartite graph is constructed by aggregating nodes in a given logmetadata graph based on factors given by service provider. By performing random walks on Factor-Item bipartite graphs constructed based on different factors, recommendations with varying semantics including collaborative filtering, context-aware collaborative filtering, and hybrid recommendation can be provided. In the following sections, we introduce two operations for constructing Factor-Item bipartite graphs and explain how to exploit the graph for recommender systems.

\subsection{Operations for Node and Edge Aggregation}

Two operations for constructing Factor-Item bipartite graphs are introduced as follows. The NE (Node and Edge)-Aggregate operation is exploited to find subgraphs of a log-metadata graph, which match a specific graph pattern, and aggregate into a simple bipartite graph. The algorithm of NE-Aggregate is described in Algorithm 1. The operation takes several input arguments. $G$ is a log-metadata graph. $A$ is an aggregate pattern which is a subgraph of $G_{S}$ that is the graph schema of the log-metadata graph $G . P$ is a pivot pair which is composed of two pivot sets $P_{1}$ and $P_{2}$, where each set is composed of node types included in the aggregate pattern $A$. Each set decides what types of nodes will be aggregated. For example, a pivot pair can be selected as $P\left(P_{1}=\{U S E R, T I M E\}, P_{2}=\{S O N G\}\right)$ when all of USER, TIME and SONG are included in the graph schema $G_{S}$. In this case, USER type nodes and SONG type nodes will become merged nodes in the result graph. We consider a pivot instance set $p_{i}$ as an instance of $P_{i}$, if every element in $p_{i}$ is a node whose type is in $P_{i}$, and every element in $P_{i}$ has a single corresponding element in $p_{i}$. For simplicity, we say a pair of pivot instance sets $\left(p_{1}, p_{2}\right)$ is an instance of $P\left(P_{1}, P_{2}\right)$ if $p_{1}$ is an instance of $P_{1}$ and if $p_{2}$ is an instance of $P_{2}$. For example, (\{'Matt', 'Night' $\},\{$ 'Song\#1'\}) is an instance of $P(\{$ USER,TIME $\},\{$ SONG $\})$, because \{'Matt', 'Night'\} is an instance of \{USER,TIME\} and \{'Song\#1'\} is an instance of $\{$ SONG\}.

Figure 3 shows an example of aggregating nodes and edges by performing NE-aggregate. If we perform NEAggregate by taking $G, A$, a pivot pair $P\left(P_{1}=\{U S E R, T I M E\}, P_{2}=\{S O N G\}\right)$, all possible instances of $P_{1}$ and $P_{2}$ are generated as nodes in the result graph $G^{\prime}$. Then, the number of subgraph instances matching the aggregate pattern and including the nodes in each pivot pair instance is assigned as edge weights between the corresponding aggregated nodes. Nodes and edges are mapped to new node and edge types $n t_{1}, n t_{2}, e t_{1}$, and $e t_{2}$. The result graph is a bipartite graph where it has two node types and two node edge types. 


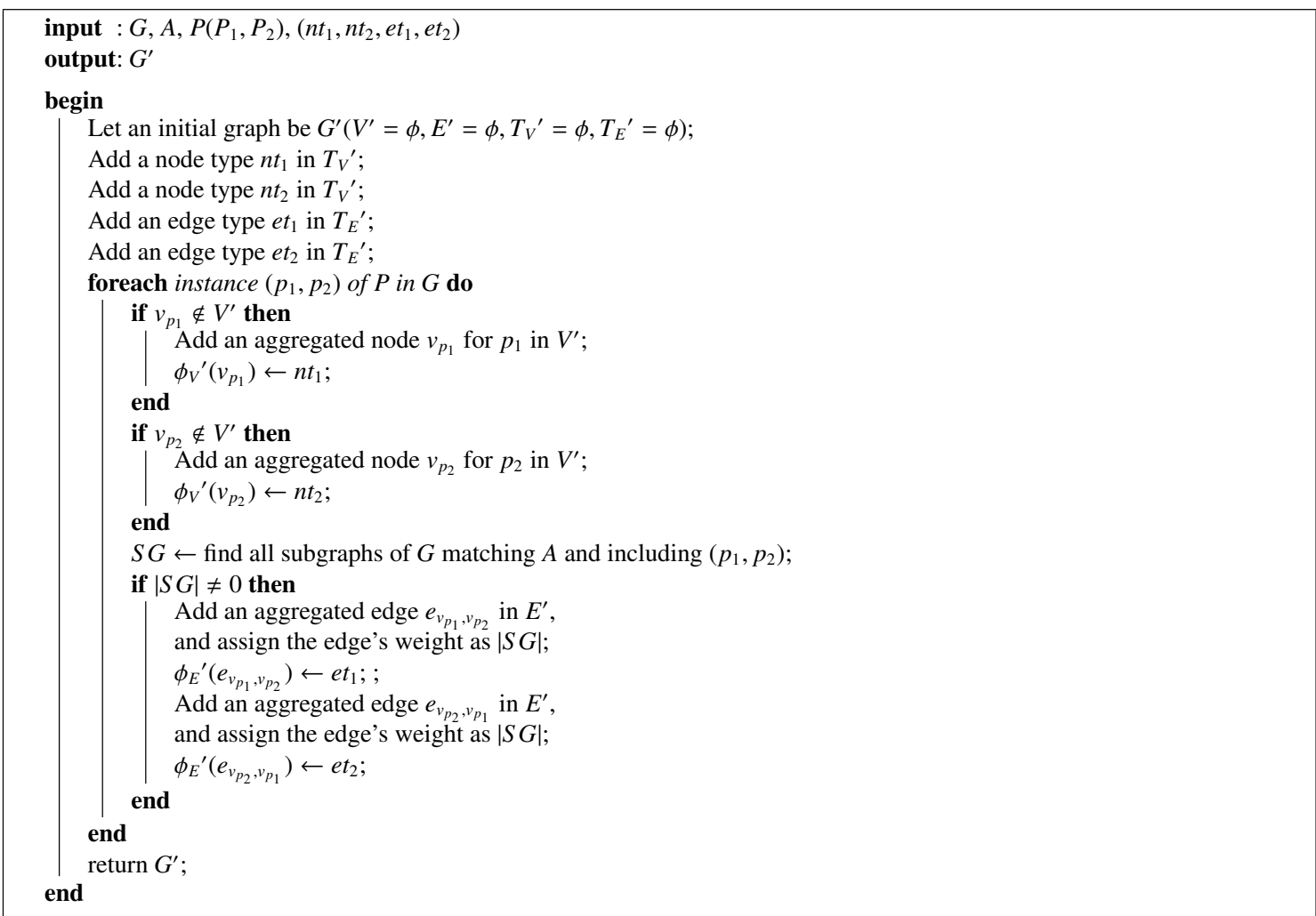

\section{Algorithm 1: NE-Aggregate}

We can achieve a summarized bipartite graph by performing NE-Aggregate on a log-metadata graph. With different inputs, various summarized bipartite graphs can be constructed. G (Graph)-Merge operation is used to merge several summarized graphs into an integrated graph. It takes a set of graphs $\Gamma=\left\{G_{1}, G_{2}, \ldots, G_{|\Gamma|}\right\}$ and results a merged graph. The result graph consists of all nodes and edges in $G_{1}, \ldots, G_{|\Gamma|}$, where the same edge with different weights are merged into an aggregated edge with a summed weight. Figure 4 shows an example of using the G-Merge operation for merging two bipartite graphs.

\subsection{Exploiting Factor-Item Bipartite Graph for Recommendation}

A Factor-Item bipartite graph has three categories of nodes that are contextual factor nodes, content factor nodes, and item nodes. Edges exist only between factor nodes and item nodes, and their weights represent the relationship strength of connected two nodes. Algorithm 3 describes the process of constructing a Factor-Item bipartite graph by aggregating nodes in a given heterogeneous graph $G$.

To construct a Factor-Item bipartite graph, several arguments for the algorithm should be set up. First of all, we should select a item node type $n t_{I}$ which represents the target node type that we want to provide for the recommendation. For example, to construct a Factor-Item bipartite graph for song recommendation using the heterogeneous graph depicted in Figure 3, the node type $S O N G$ should be selected as $n t_{I}$. Second, we need to select a node type $n t_{T}$ which describes events about the selected $n t_{I}$. For instance, the node type PLAY can be selected as $n t_{T}$ when $n t_{I}$ is $S O N G$. Last, a contextual factor set $F_{C}=\left\{c f_{1}, c f_{2}, \ldots, c f_{\left|F_{C}\right|}\right\} \subseteq F_{C}{ }^{\Sigma}$ and a content factor set $F_{T}=\left\{t f_{1}, t f_{2}, \ldots, t f_{\left|F_{T}\right|}\right\} \subseteq F_{T}{ }^{\Sigma}$ are needed to be selected by service providers based on their domain knowledge. $F_{C}{ }^{\Sigma}$ and $F_{T}{ }^{\Sigma}$ are the complete factor sets which are composed of all possible factors defined for the selected $n t_{I}$ and $n t_{T}$. A contextual factor can be defined as a set whose elements are the node types connected to $n t_{T}$ (except $n t_{I}$ ). For example, when $n t_{I}$ is $S O N G$ and $n t_{T}$ is 


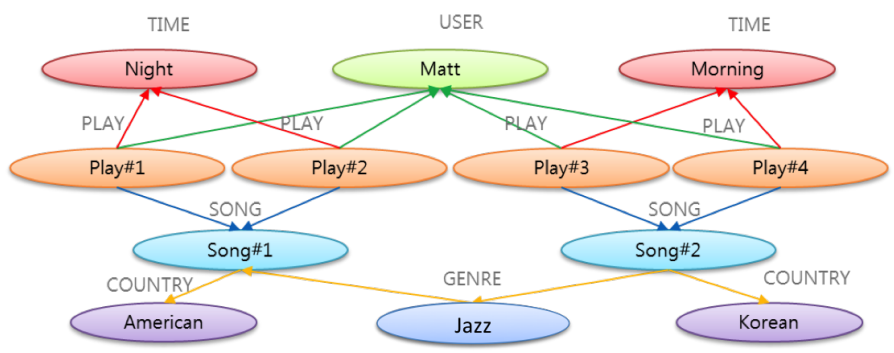

Heterogeneous Graph $G$

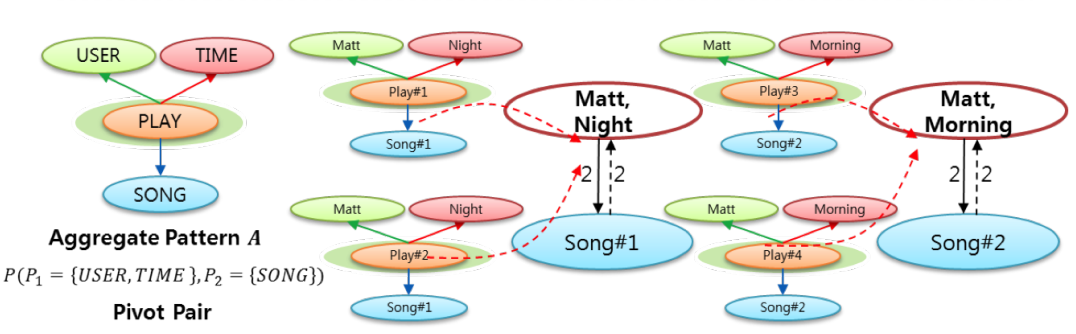

Figure 3. Aggregating nodes and edges by using NE-Aggregate

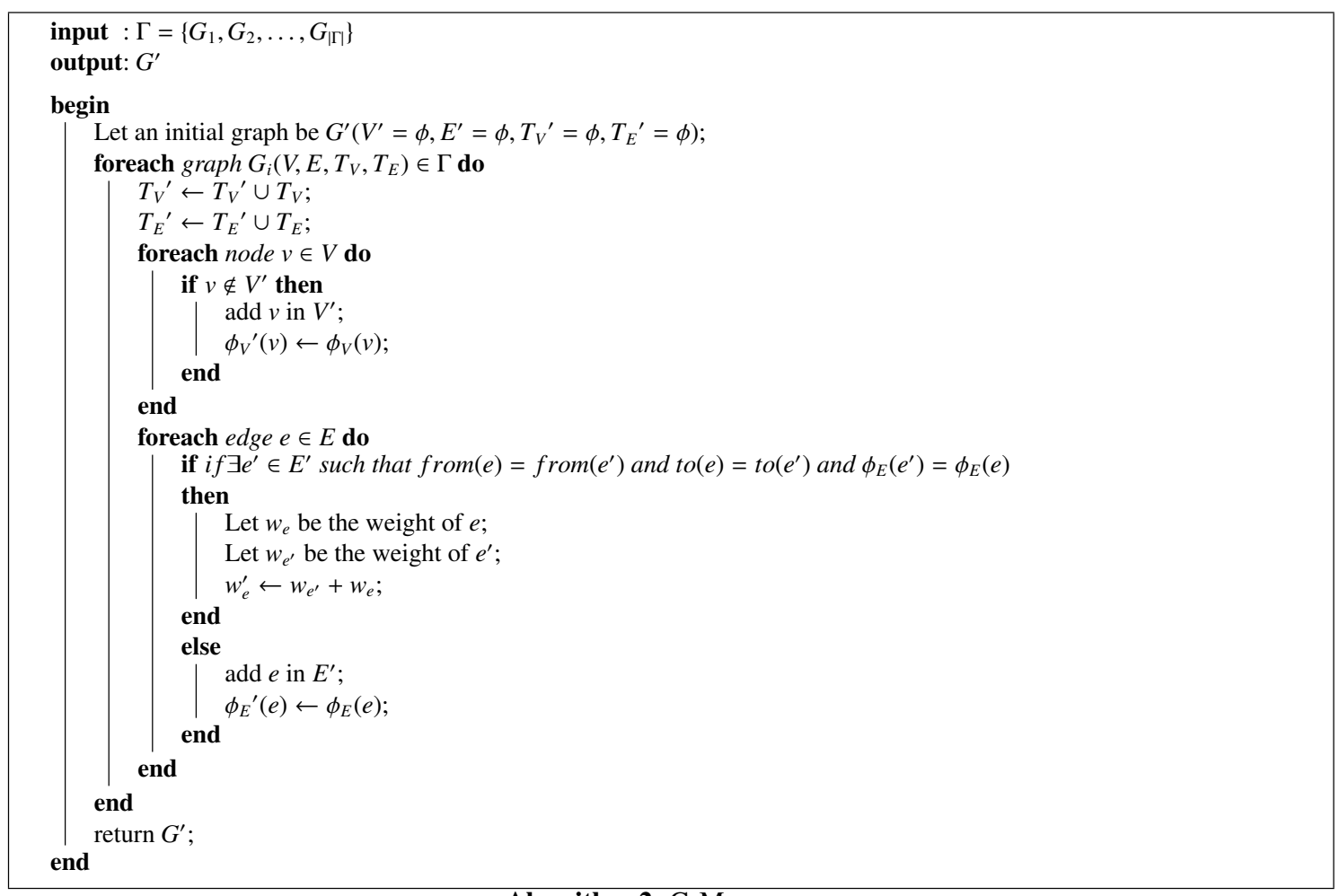

Algorithm 2: G-Merge 


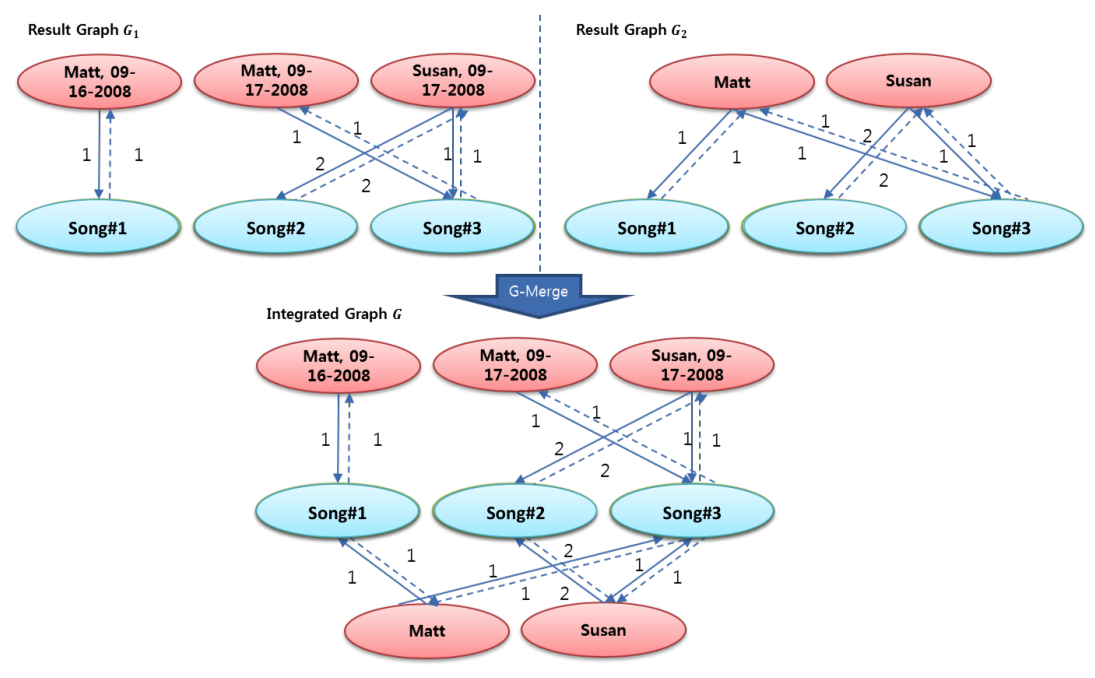

Figure 4. Merging graphs by performing $G$-Merge operation

$P L A Y$, the complete contextual factor set is defined as $F_{C}{ }^{\Sigma}=\left\{c f_{1}=\{U S E R\}, c f_{2}=\{T I M E\}, c f_{3}=\{U S E R, T I M E\}\right\}$. Similarly, a content factor can be defined as a set, where its elements are the node types that are linked to $n t_{I}\left(\right.$ except $\left.n t_{T}\right)$. So, when the node type $S O N G$ is selected as $n t_{I}$, the complete content factor set is defined as $F_{T}{ }^{\Sigma}=\left\{t f_{1}=\{C O U N T R Y\}\right.$, $\left.t f_{2}=\{G E N R E\}, t f_{3}=\{C O U N T R Y, G E N R E\}\right\}$.

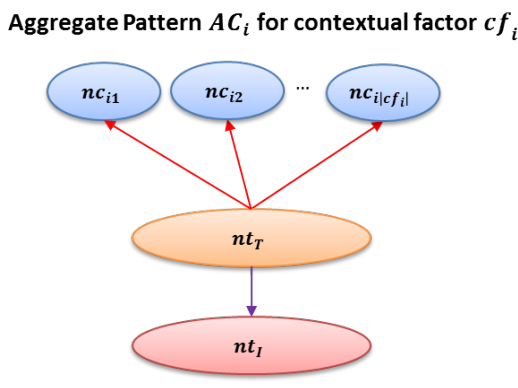

Aggregate Pattern $A T_{i}$ for content factor $t f_{i}$

Figure 5. Aggregate patterns for constructing Factor-Item bipartite graphs

Aggregate patterns are required for constructing a Factor-Item bipartite graph using the given factors. For a contextual factor $c f_{i}=\left\{n c_{i 1}, n c_{i 2}, \ldots, n c_{i\left|c f_{i}\right|}\right\}$ and a content factor $t f_{i}=\left\{n t_{i 1}, n t_{i 2}, \ldots, n t_{i\left|t f_{i}\right|}\right\}$, we use the aggregate patterns depicted in Figure 5 for constructing a Factor-Item bipartite graph. These two aggregate patterns are derived from the most common subgraph patterns that repetitively appear in the log-metadata graph constructed in the way we explained in the Section 4.1. A subgraph shaped in the first pattern is created for every tuple in the original source log table. For example, let assume that the schema of a music log table is PLAY(ID, USER, SONG, TIME). Then, every tuple in the log table identified the ID will be transformed into a PLAY type node, and the node will be linked with nodes whose types are USER, TIME, and SONG. In general, the number of $\operatorname{logs}$ in a log table is huge, as logs are continuously accumulated. Similarly, subgraphs with the second pattern are created for tuples in the source metadata table. A metadata table for a type of entities (e.g., SONG) is usually composed of several columns describing each entitys property. For example, let us assume that the schema of a metadata table for movies is MOVIE (ID, TITLE, COMPOSER, GENRE), and the columns COMPOSER and GENRE have foreign-key relationships with the respective COMPOSER and GENRE tables. In this case, every tuple in the MOVIE table will be converted 


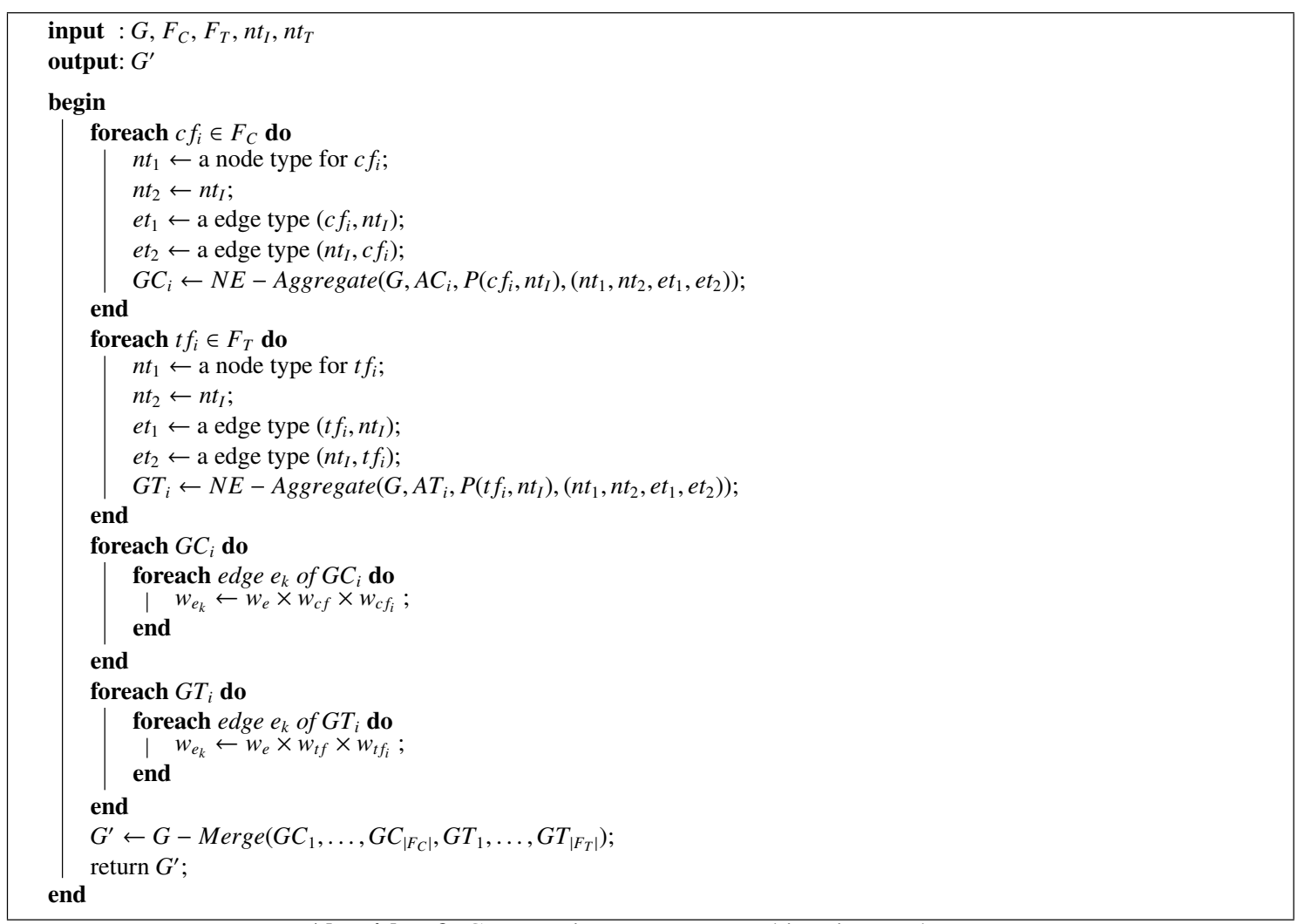

Algorithm 3: Constructing a Factor-Item bipartite graph

into SONG nodes and the nodes will be linked with their corresponding COMPOSER and GENRE type nodes. It is expected that we can significantly reduce the number of nodes and edges using the patterns. We assume that the weight $w_{c f_{i}}$ for each contextual factor $c f_{i}$ is given, where $w_{c f_{1}}+w_{c f_{2}}+\ldots+w_{c f_{\mid F C l}}=1$. Each $w_{c f_{i}}$ decides how much the contextual factor will affect the recommendation results. Similarly, weight $w_{t f_{i}}$ is given for each content factor $t f_{i}$, where $w_{t f_{1}}+w_{t f_{2}}+\ldots+w_{t f_{\left|F_{T}\right|}}=1$. We also assume that the weights $w_{c f}$ and $w_{t f}$ are given. The $w_{c f}$ is the weight of contextual factors, and the $w_{t f}$ is the weight of content factors, where $w_{c f}+w_{t f}=1$.

The Figure 6 shows an example of a constructed Factor-Item bipartite graph when a contextual factor set $F_{C}=$ $\{\{U S E R\},\{U S E R, T I M E\}\}$ and a content factor set $F_{T}=\{\{C O U N T R Y\},\{G E N R E\}\}$ are given, where $n t_{I}$ is $S O N G$ and $n t_{T}$ is $P L A Y$.

In order to define a utility function, we adopt Personalized PageRank algorithm (Haveliwala, 2003). The PageRank score of a node will get higher score when the node is connected to more important nodes with less outgoing links. By using the measure, we can take advantages of propagation and attenuation properties that are introduced in Gori et al.(Gori and Pucci, 2007). The propagation property is that the relevance of the nodes propagates following the links, and the attenuation property is that the propagation strength decreases as the propagation goes further from the starting node. For example, a node $n_{1}$ is linked to a node $n_{2}$, and another node $n_{3}$ is related to the $n_{2}$, then the nodes $n_{1}$ and $n_{3}$ are related to each other but less related than directly linked nodes. By using these properties, we can measure the relevance between nodes, although they are not directly linked.

First of all, we derive a $|V| \times|V|$ adjacency matrix $M$ for a Factor-Item bipartite graph $G=\left(V, E, T_{V}, T_{E}\right)$ as follows:

$$
M(i, j)= \begin{cases}w_{k} & , \text { if } \exists e_{k}\left(v_{i} \rightarrow v_{j}\right) \in E \\ 0 & , \text { otherwise }\end{cases}
$$




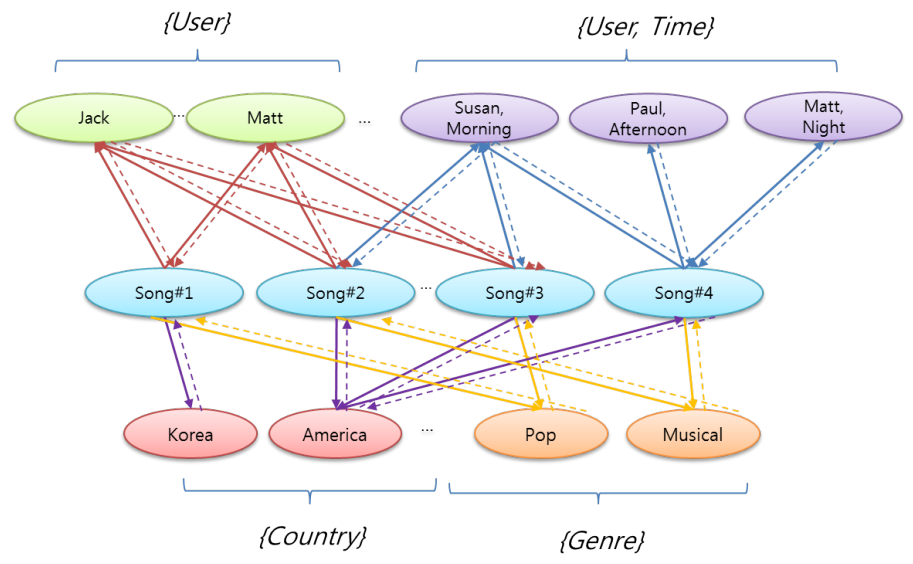

Figure 6. An example of a constructed Factor-Item bipartite graph

where $w_{k}$ is the weight of an edge $e_{k}\left(v_{i} \rightarrow v_{j}\right)$ from $v_{i}$ to $v_{j}$, and $M(i, j)$ is the $M$ 's element of the $i$-th row and the $j$-th column. There can be only one aggregated edge between two nodes for each direction in a Factor-Item bipartite graph. So, we do not need to sum up the weights of multiple edges existing between two nodes.

Random walks on a heterogeneous graph can be characterized by a transition matrix $P$ which can be computed by normalizing the matrix $M$ as follows:

$$
P(i, j)= \begin{cases}\frac{M(i, j)}{\sum_{\left.v_{k} \in \operatorname{Eotlink}_{i}\right]}^{M(i, k)}} & , \text { if outlink }\left[v_{i}\right] \neq \phi \\ 0 & , \text { otherwise }\end{cases}
$$

where $P(i, j)$ is the $P$ 's element of the $i$-th row and the $j$-th column, and $M(i, j)$ is the $M$ 's element of the $i$-th row and the $j$-th column.

PageRank can be computed by the following equation $\vec{r}=c P^{T} \vec{r}+(1-c) \frac{1}{n} \vec{e}$, where $n$ is the number of nodes, $r_{i}$ is the rank score for node $v_{i}, \vec{e}=(1,1, \ldots, 1)^{T}$, and $c$ is a damping factor constant that is normally given as 0.85 . In this work, we use a query vector $\vec{q}$ as the teleport vector instead of using the uniform teleport vector $\frac{1}{n} \vec{e}$. As we explained in section 3, a query $Q$ for a graph-based recommendation is defined as $Q=(N, \tau, k)$. We consider that the current context of a user represented by a set of entities $E_{q}=\left\{e_{1}, e_{2}, \ldots, e_{\left|E_{q}\right|}\right\}$ is used to formulate a recommendation query. In a Factor-Item bipartite graph, a node can represent more than one entity. We use a query node set $N \subseteq V$, where $N$ is composed of the nodes representing the entities in $E_{q}$ or the nodes representing the combinations of entities in $E_{q}$. Thus, we define a vector $\breve{\vec{q}}$ as follows:

$$
\breve{q}_{i}= \begin{cases}1 & , \text { if } q_{i} \subseteq N \\ 0 & , \text { otherwise }\end{cases}
$$

By normalizing the vector $\breve{\vec{q}}$, we compute a query vector $\vec{q}$ whose elements sum to 1 . Then, we use the normalized vector $\vec{q}$ as the teleport vector for ranking nodes in the graph. So, the ranking score vector is calculated as $\vec{r}=$ $c P^{T} \vec{r}+(1-c) \vec{q}$. We can use the $r_{i}$ which is the i-th element of $\vec{r}$ as the ranking score for each node $v_{i}$ so that we can sort the nodes whose node types are $\tau$ and find top-k nodes for the query.

Depending on needs, service providers can construct different Factor-Item bipartite graphs by selecting various contextual and content factors. Transforming a heterogeneous graph into a Factor-Item bipartite graph can reduce the graph size significantly, but it could lead to some information loss, thus selecting factors should be done carefully. Providing recommendations by exploiting a Factor-Item bipartite graph composed of contextual factors and content factors depicted in Figure 6 can be considered as a hybrid recommendation method which combines collaborative filtering, content-based filtering, and context-aware collaborative filtering. However, we can use simpler Factor-Item 
bipartite graph structures. Figure 7(a) shows an example of a Factor-Item bipartite graph for processing collaborative filtering $\left(F_{C}=\{\{U S E R\}\}, n t_{I}=S O N G, n t_{T}\right.$ is $\left.P L A Y\right)$. Figure $7(b)$ shows an example of a Factor-Item bipartite graph for context-aware collaborative filtering. $\left(F_{C}=\{\{U S E R, T I M E\}\}, n t_{I}=S O N G, n t_{T}\right.$ is $\left.P L A Y\right)$.

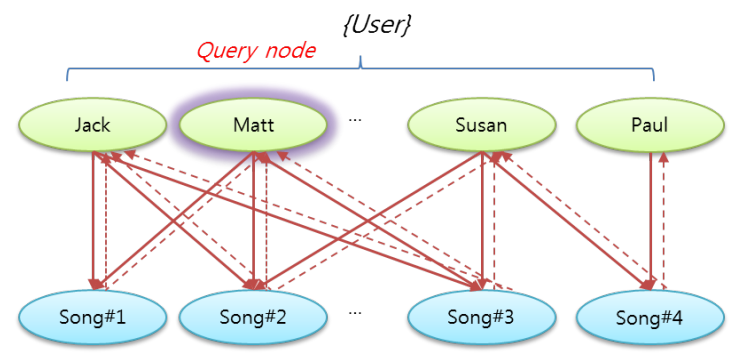

(a) A Factor-Item bipartite graph for collaborative filtering

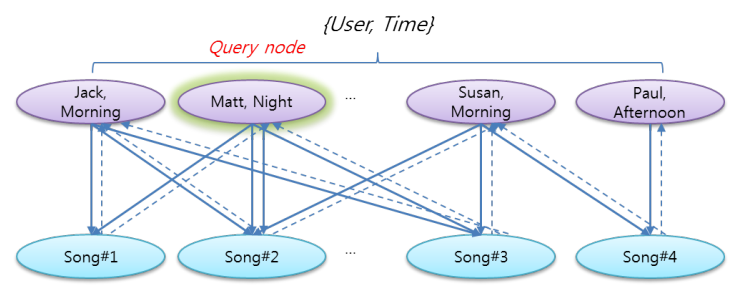

(b) A Factor-Item bipartite graph for context-aware collaborative filtering

Figure 7. Variations of Factor-Item bipartite graphs

\section{Experiments}

Although the initial aim of our research is to achieve flexibility and capability of exploiting rich information for recommendation, we should not give up the recommendation performance in terms of accuracy. So, we conducted several experiments for evaluating our method. The first experiment is to evaluate the recommendation performance of the typical recommendation case, which is recommending items to users. Then, the second experiment is designed to look at the effects on exploiting contextual factors. In the third experiment, we evaluate the performance of hybrid recommendation by varying the weight of $w_{c f}$ and $w_{t f}$. In the last experiment, we test how much we can reduce the graph size without losing the recommendation accuracy. In the experiments, we used two different datasets for testing two recommendation scenarios. The first scenario is a typical case that the recommender system recommends previously unseen items of the users. This scenario is suitable when dealing with contents that are not repeatedly consumed by users. For this scenario, we used the LG Oz Store dataset (OZSTORE) in that users do not purchase the same applications repetitively. The second scenario is that the system recommends items to user including the items that have been already consumed by the user. We used the last.fm dataset (LASTFM) for this case because users generally listen to the same songs repeatedly. For the first scenario, we only sort the applications that have never been purchased by the given user, and we sort all songs to generate the predicted ranking list for the second one. Detailed experimental settings are explained in the following section.

\subsection{Experimental Setup}

For our experiments, we used two real-world datasets. The first dataset consists of logs from a popular online music streaming service called last.fm. The dataset contains 673,293 music listening logs of 1,699 users on 3,000 songs from 01-01-2008 to 11-22-2008. The other dataset consists of logs from a mobile application market called $L G O z$ Store. It contains 862,291 application purchase logs of 4,000 users on 4,000 applications from 06-06-2010 
to 04-26-2011. For both datasets, the original logs only contain USER, ITEM, and DATE attributes. Therefore, we added several contextual attributes such as DOW (day of week), HALF (the value 1 means the first six month of the year, and the value 2 means the second six month of the year), QUARTER (each value 1,2,3,4 means the 1st, 2nd,3rd, and 4th quarter of the year) by analyzing the DATE attribute. For the second dataset, there exist a metadata table which describe items in the log table. The metadata table contains four dimensions that are item_id, comp_name (e.g., Samsung, LG), platform (e.g., Android, Windows Mobile), app_category (e.g., Game, Utility, Camera).

For each dataset, we sorted the logs into ascending order by DATE and used the first $80 \%$ of the full data as the training set, and we generated test sets from the rest of the data. Each test set consists of randomly chosen 1,000 logs. Generally, the rating error measures such as Mean Absolute Error (MAE) or Root Mean Square Error (RMSE) have been used to evaluate recommender systems that focus on rating estimation. However, due to the fact that we do not focus on estimating actual ratings of users and only focus on top-k recommendation of entities, we cannot use such metrics. Therefore, we use a metric called Hit Rate HR@k (Lee et al., 2010) that is more suitable for measuring the performance of top-k recommendation. We can consider our problem as the problem of predicting hidden entities in test cases.HR@ $k$ is defined as (\#hit)/n, where $\mathrm{n}$ is the number of tests and \#hit is the number of cases that the hidden entity in the test case is ranked in top-k in the produced ranking list by a recommender system.

Each dataset is transformed into a heterogeneous graph as explained in Section 4. Figure 8 shows the schemas of the graphs constructed from our experimental datasets.

Personalized PageRank computation plays an important role to generate the recommendation results. There exist many available tools that support the Personalized PageRank computation such as Pregel (Malewicz et al., 2010), GraphX (Xin et al., 2013), GraphChi (Kyrola et al., 2012), TurboGraph Han et al. (2013), NetworkX (Hagberg et al., 2008) and etc. However, for our experiment, we implemented our own Personalized PageRank computation module using Java for later extension. As explained in Section 4.2 and Section 5.2, a graph can be represented by a $N \times N$ transition matrix, where $N$ is the number of nodes in the graph. However, storing a graph using $N \times N$ multidimensional array is not feasible when the graph is large, since its space complexity is $O\left(N^{2}\right)$. For instance, when $N$ is 100000 , then we need $100000^{2} \times 4$ bytes (that is $37.25 \mathrm{~GB}$ ) of reserved memory space to store the graph. To achieve scalability, we implemented a sparse matrix library using HashMap that stores a matrix as a set of key value pairs $\{(i, j), w\}$, where $i$ and $j$ are the respective row and column indices and $w$ is the value of the corresponding matrix element. Note that the space complexity of this approach is $O(n)$. Using this library, we could load the entire graph into memory for faster matrix-vector multiplication that is the core computation of Personalized PageRank computation. We validated our implementation of Personalized PageRank by comparing the results with the results of NetworkX.



(a) LG Oz Store dataset

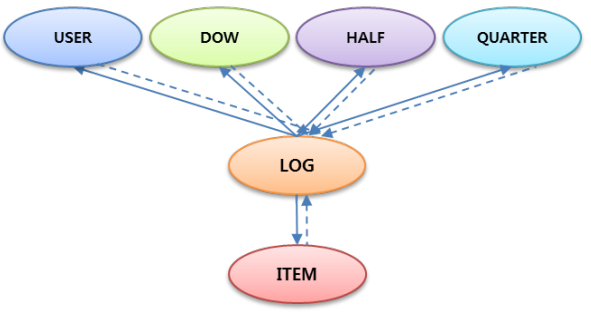

(b) last.fm dataset

Figure 8. Schemas of the graphs constructed for our experiments

\subsection{Baseline Methods}

We compared the top-k recommendation performance of our method with several existing methods: Popularitybased (POP), User-based k-Nearest Neighborhood CF (UKNN), ItemRank (IRANK), PureSVD (PSVD), and Nä̈ve Graph-based Recommendation Method (NAÏVE). Because UKNN, IRANK, PSVD are designed for two dimensional User-Item matrix, we transformed our training dataset into a USER $\times I T E M$ pseudo rating matrix by considering the normalized access count of a user on an item as the users pseudo rating on the item.

Popularity-based (POP): Popularity-based method generates a ranking list based on the popularity of items in the training dataset. The method is not a personalized method, thus it generates the same ranking list for every user. 
k-Nearest Neighbor User-based CF (UKNN): k-nearest neighbor user-based CF finds the k similar users to the active user and aggregates the ratings of the similar users. We used the aggregation function as follows:

$$
\vec{r}_{u, i}=\overline{r_{u}}+\frac{\Sigma_{u^{\prime} \in \hat{U}} \operatorname{sim}\left(u, u^{\prime}\right) \times\left(r_{u^{\prime}, i}-\overline{r_{u^{\prime}}}\right)}{\Sigma_{u^{\prime} \in \hat{U}} \operatorname{sim}\left(u, u^{\prime}\right)}
$$

where $r_{u, i}$ is the rating of user $u$ on item $i, \overline{r_{u}}$ is the average ratings of user, $\hat{U}$ is the set of $k$ most similar users, and the $\operatorname{sim}\left(u, u^{\prime}\right)$ is the cosine similarity of rating vectors of $u$ and $u^{\prime}$. We empirically set $k=70$ for our experiments. The estimated ratings of the active user are used as the rank score for top-k recommendation.

ItemRank (IRANK): ItemRank (Gori and Pucci, 2007) firstly creates an item graph based on the given rating matrix. The number of users who both consumed the items are assigned to corresponding edge weights. Then, it computes the Personalized PageRank score of each item on the constructed graph by using a teleport vector that is constructed from the active user's previous ratings.

PureSVD (PSVD): PureSVD (Cremonesi et al., 2010) is one of the state-of-the art methods and known to perform very well for top-k recommendation, although the method cannot be used to estimate actual ratings of users. It is based on matrix factorization that reduces the dimension of the matrix, so the number of dimensions d should be decided. We empirically set the $d=30$ for our experiments.

Naive Graph-based Recommendation (Naive): It is the graph-based recommendation method explained in Section 3. We firstly model the base dataset as a heterogeneous graph. Then, the Personalized PageRank score of each node for a recommendation query is utilized as node ranking score.

\subsection{Performance of Collaborative Filtering}

In this section, we test the collaborative filtering performance of the proposed recommendation method based on Factor-Item bipartite graph. We constructed a Factor-Item bipartite graph by summarizing the heterogeneous graph modeled from each dataset, where $F_{C}=\left\{c f_{1}=\{U S E R\}\right\}, F_{T}=\phi, n t_{T}$ is $L O G$ and the $n t_{I}$ is ITEM. Each user appears in each tuple of test set can be considered as an active user. Then, we predict the rank of the item appears in the tuple and measure the performance in terms of $H R @ k$. We used $Q=\left\{N=\left\{n_{\text {user }}\right\}, \tau=I T E M, k\right\}$ as a query for each test case, where $n_{\text {user }}$ is the node that corresponds to the active user. Figure 9 shows the performance of our method with this configuration. We used the term FIB-CF as an abbreviation of the proposed method. For the first case, which is the case of recommending previously unseen items to users using OZSTORE dataset, NAIVE shows the best performance. We can infer that it is because NAIVE could take advantages of rich information such as items' metadata. Our method outperforms POP, UKNN, and IRANK, and it shows the competitive performance with PSVD. For the LASTFM case, our method outperforms all of the baseline methods that we compared. We can infer that exploiting indirect relationships among entities through random walk process can resolve the data sparsity and contribute to the recommendation accuracy. Although only the first scenario has been considered in many previous works, the second scenario is also very important because there are many applications dealing with repeatedly consumed items (e.g., music, food). In the case of recommending items repeatedly, not only what item to recommend but also in what situation to recommend is important. Note that our method has advantage in that it can be easily extended to incorporate various other contextual attributes (e.g. location, time) into recommendation. We discuss the effects on incorporating rich information into our proposed method in the following section.

\subsection{Performance of Context-aware Collaborative Filtering}

In this experiment, we test context-aware collaborative filtering performance of our method with various contextual factor settings. Each test case (a tuple) can be considered as a set of entities. We hide the target item in each tuple and use the other entities in the tuple to compose a query for each test. Using the query, we predict the rank of the hidden target entity. Note that we do not use any content factors, thus $w_{c f}$ is set to 1 and $w_{t f}$ is set to 0 . Table 1 describes our experimental setting variations, and Table 2 shows the experimental result. We can consider the $F_{1}$ as the setup for performing collaborative filtering. $F_{2}$ is a setting for recommending items to users based on contextual information without using user information. We can consider $F_{3}$ and $F_{4}$ as the cases of utilizing user preferences depending on a specific dimension. From $F_{5}$ to $F_{7}$ are the examples of merging several factors to produce ranking list. For example, $F_{5}$ can be considered as the case of merging users general preference and users preference depending on HALF 


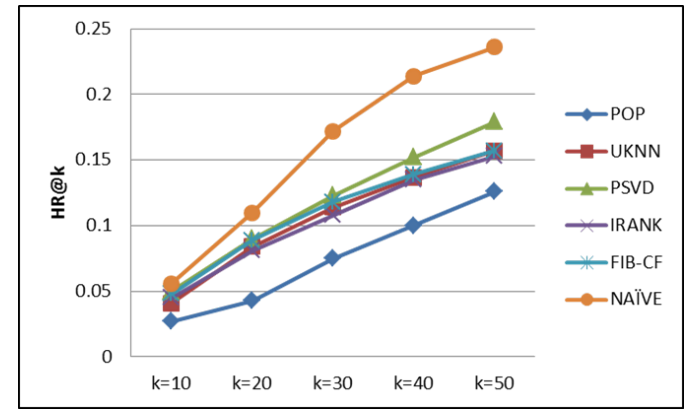

(a) LG Oz Store dataset

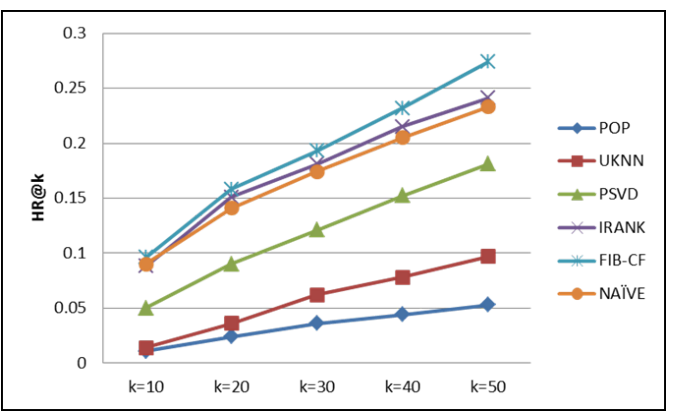

(b) last.fm dataset

Figure 9. Performance of collaborative filtering

Table 1. Contextual factor settings

\begin{tabular}{|c|c|}
\hline Factor Set & Weight \\
\hline$F_{1}=\left\{f_{1}=\{U S E R\}\right\}$ & $w_{1}=1.0$ \\
\hline$F_{2}=\left\{f_{1}=\{D O W\}\right\}$ & $w_{1}=1.0$ \\
\hline$F_{3}=\left\{f_{1}=\{U S E R, H A L F\}\right\}$ & $w_{1}=1.0$ \\
\hline$F_{4}=\left\{f_{1}=\{U S E R, Q U A R T E R\}\right\}$ & $w_{1}=1.0$ \\
\hline$F_{5}=\left\{\begin{array}{c}f_{1}=\{U S E R\}, \\
f_{2}=\{U S E R, H A L F\}\end{array}\right\}$ & $\begin{array}{l}w_{1}=0.2 \\
w_{2}=0.8\end{array}$ \\
\hline$F_{6}=\left\{\begin{array}{c}f_{1}=\{U S E R\} \\
f_{2}=\{U S E R, H A L F\} \\
f_{3}=\{U S E R, Q U A R T E R\}\end{array}\right\}$ & $\begin{array}{l}w_{1}=0.2 \\
w_{2}=0.4 \\
w_{3}=0.4\end{array}$ \\
\hline$F_{7}=\left\{\begin{array}{c}f_{1}=\{U S E R\}, \\
f_{2}=\{\text { HALF }\}, \\
f_{3}=\{\text { DOW }\}, \\
f_{4}=\{\text { QUARTER }\}, \\
f_{5}=\{\text { USER, DOW }\}, \\
f_{6}=\{\text { USER, HALF }\}, \\
f_{7}=\{U S E R, Q U A R T E R\}\end{array}\right\}$ & $\begin{array}{l}w_{1}=0.15 \\
w_{2}=0.05 \\
w_{3}=0.05 \\
w_{4}=0.05 \\
w_{5}=0.1 \\
w_{6}=0.3 \\
w_{7}=0.3\end{array}$ \\
\hline
\end{tabular}

Table 2. Performance of context-aware collaborative filtering

\begin{tabular}{|c|c|c|c|c|c|c|c|c|c|c|c|c|c|c|}
\hline HR@k & \multicolumn{7}{|c|}{ SCENARIO 1 (OZSTORE) } & \multicolumn{7}{|c|}{ SCENARIO 2 (LASTFM) } \\
\hline HR@10 & 0.048 & 0.028 & 0.008 & 0.008 & 0.058 & 0.062 & 0.057 & 0.096 & 0.015 & 0.098 & 0.024 & 0.097 & 0.099 & 0.095 \\
\hline HR@20 & 0.089 & 0.060 & 0.022 & 0.018 & 0.100 & 0.103 & 0.095 & 0.158 & 0.024 & 0.160 & 0.037 & 0.164 & 0.164 & 0.169 \\
\hline HR@30 & 0.118 & 0.091 & 0.039 & 0.031 & 0.125 & 0.130 & 0.135 & 0.193 & 0.039 & 0.208 & 0.044 & 0.210 & 0.210 & 0.209 \\
\hline HR@50 & 0.157 & 0.138 & 0.091 & 0.070 & 0.195 & 0.203 & 0.223 & 0.274 & 0.053 & 0.294 & 0.062 & 0.291 & 0.292 & 0.270 \\
\hline
\end{tabular}

dimension. Note that there are virtually infinite settings of utilizing contextual information. Our method has such flexibility so that recommendation service providers can setup their recommendation to reflect their requirements.

As we can see the result of $F_{2}$, recommendation performance decreases when our method does not utilize user 
information. It is reasonable that user preference is critical for recommendation. For some cases such as $F_{3}, F_{4}(\mathrm{OZS}$ TORE) and $F_{4}$ (LASTFM), although our method utilizes the combination of USER and other contextual information, the recommendation performances decrease. It is because the system often cannot make a recommendation list when there is no matched node for given queries. We can consider $F_{5}, F_{6}$, and $F_{7}$ as the solution for this problem, these three settings allow merging the factors so that it resolves the node matching problem. As results, they show better performance than the baseline $\left(F_{1}\right)$. Note that our method provides better accuracy than PSVD for OZSTORE dataset by using contextual factors, although it shows similar performance in the previous experiment. The experimental result shows that our method can improve the recommendation performance by exploiting various contextual information. Although there can be other contextual settings that can bring on better recommendation performances, we do not focus on this issue here. Another advantage is that it can increase the diversity of recommendation results, because the system produces different ranking results not only to different users but also to different context. Even when the system recommends items to the same user, if the additional nodes (e.g. 'Seoul', 'Summer', and etc.) are used as query nodes, different ranking lists will be produced by the method.

\subsection{Performance of Hybrid Recommendation}

Figure 10. Performance of hybrid recommendation (OZSTORE)

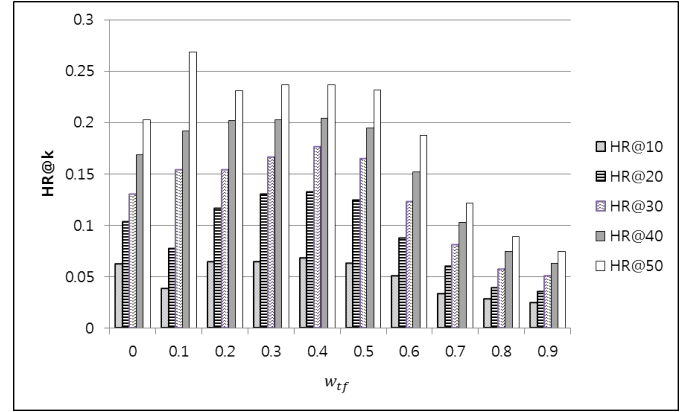

(a) Impact of varying $w_{t f}$ on $H R @ k$

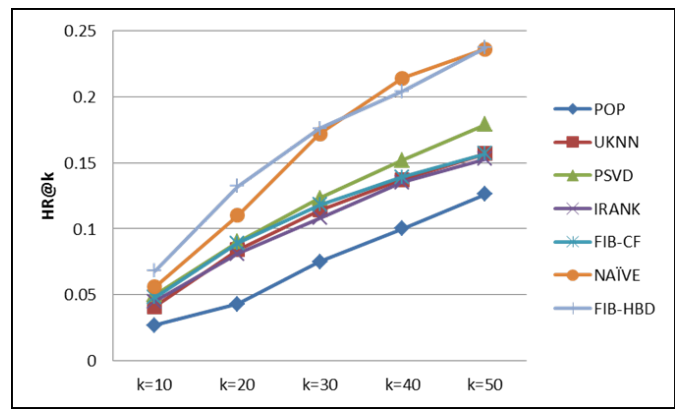

(b) Comparison with the other approaches

For the OZSTORE dataset, we can define and take advantages of content factors, because ITEM nodes are described by several other types of node. We used the contextual factor setting $F_{7}$ which showed the best results among the context-aware collaborative filtering settings. Additional, we set up a content factor set $F_{T}=\left\{\left\{C O M P \_N A M E\right\}\right.$, $\left.\left\{A P P \_C A T E G O R Y\right\},\{P L A T F O R M\}\right\}$. We assign the same weight $\frac{1}{3}$ to every content factor. We tested the impact on performance HR@k by varying the weight $w_{t f}$ from 0.0 to 0.9 . When $w_{t f}$ is set to 0.0 , it can be considered as context-aware collaborative filtering without exploiting content factors. As the $w_{t f}$ increases, the impact of content factors increases. Figure 10(a) shows the results. When $w_{t f}$ is set to $0.1, H R @ k$ slightly decreased at $k=10$ and $k=20$; however HR@ $k$ increased at $k=30, k=40$, and $k=50$. As the $w_{t f}$ increases, the HR@ $k$ increases until $w_{t f}=0.4$ then decreases. The Figure 10(b) shows the best case performance comparison of hybrid recommendation using Factor-Item bipartite graph (FIB-HBD) with the other approaches. FIB-HBD shows the best performance among all baseline methods including Factor-Item bipartite graph-based collaborative filtering (FIB-CF) and context-aware collaborative filtering (FIB-CACF). We notice that exploiting context and content factors at the same time can significantly increase the recommendation performance; however, proper $w_{t f}$ should be selected for achieving the best results.

\subsection{Comparing with the Nä̈ve Method}

In order to evaluate how much we can reduce the graph size by aggregating nodes in a heterogeneous graph and see the impact of node aggregation, we compared the constructed graph size and the performance of three different FIB-based recommendation methods with the naïve graph-based recommendation method. The performance of FIBHBD is only measured for the OZSTORE dataset, because metadata is not available for the LASTFM dataset. Table 
Table 3. Performance and graph size comparison with the naïve method

\begin{tabular}{|c|c|c|c|c|c|c|c|}
\hline \multirow{2}{*}{ HR@k } & \multicolumn{4}{|c|}{ SCENARIO 1 (OZSTORE) } & \multicolumn{3}{c|}{ SCENARIO 2 (LASTFM) } \\
\cline { 2 - 8 } & NAÏVE & FIB-HBD & FIB-CACF & FIB-CF & NAÏVE & FIB-CACF & FIB-CF \\
\hline \#edge & 6924290 & 2020226 & 2000516 & 666810 & 5337310 & 918500 & 243842 \\
\hline \#node & 708438 & 24135 & 23685 & 7199 & 537337 & 6638 & 3594 \\
\hline HR@10 & 0.056 & 0.068 & 0.062 & 0.048 & 0.090 & 0.099 & 0.096 \\
\hline HR@20 & 0.110 & 0.132 & 0.103 & 0.089 & 0.141 & 0.164 & 0.158 \\
\hline HR@30 & 0.172 & 0.176 & 0.13 & 0.118 & 0.174 & 0.21 & 0.193 \\
\hline HR@40 & 0.214 & 0.204 & 0.169 & 0.139 & 0.205 & 0.249 & 0.232 \\
\hline HR@50 & 0.236 & 0.237 & 0.203 & 0.157 & 0.233 & 0.292 & 0.274 \\
\hline
\end{tabular}

3 shows the experimental results. In the case of OZSTORE dataset, FIB-HBD shows the best performance on $H R @ k$. NAÏVE shows slightly lower performance than FIB-HBD, but it shows better performance at $k=40$ and $k=50$. Note that $H R @ k$ is generally more important at $k$ is a small number. FIB-CACF shows better performance than NAÏVE at $k=10$, but FIB-CACF and FIB-CF generally show worse performance than NAÏVE approach. We can infer that it is because FIB-CACF and FIB-CF do not take advantages of metadata of items. Compared to the graph exploited for NAÏVE, Factor-Item graphs are significantly smaller. In the case of OZSTORE dataset, FIB-HBD shows the best performance, although the number of edges in the graph exploited for FIB-HBD is decreased by about 70\%. NAÏVE shows the worst performance among the tested methods in the case of LASTFM dataset, although it exploits the largest graph. It implies that exploiting large size of graph does not guarantee the recommendation performance. Compared to NAÏVE, we found that FIB-CACF shows 10\% increased HR@ $k$ at $k=10$, although the number of edges are decreased by about $80 \%$. In graph-based recommender systems, the sizes of exploited graphs are critical for achieving performance in terms of processing time. The experimental results show that summarizing graphs into Factor-Item bipartite graph can construct more effective and concise graph compared to the graph exploited by NAÏVE.

\subsection{Discussion}

There are many parameters for creating Factor-Item bipartite graph, and it might be challenging for service providers to do it from scratch. Based on our experience, we made the following guideline for service providers to choose effective factors and their weights.

First of all, in most cases, 'user is the most important factor, as recommendation is mainly a procedure of personalization. Unless user information is not available, it is always recommended to select user identification as the main factor when creating Factor-Item bipartite graph. Second, service providers need to list up all available contextual factors. We noticed that using more number of factors do not always provide better accuracy but increase the size of constructed graph. Thus, we recommend service providers to select a small number (one to three) of contextual factors by speculating a users item consumption patterns based on domain knowledge. For example, let us assume that you are a service provider of a news recommender system. You could think of item consumption patterns, such as a user might prefer to read stock market report in the morning, or the user may prefer to read movie reviews and blogs on weekends. This speculated patterns imply that the \{USER, DOW (Day of Week) $\}$ and $\{$ USER, TOD (Time of Day) $\}$ are potentially important of factors. The weights of each factor can be given as the same values at first. Then, you need to test if adding each potentially important factor to the user identification factor is helpful to improve recommendation quality. Only the contextual factors that improve the recommendation quality are selected and the same weights are initially given to all factors. Then, testing the recommendation performance with increasing or decreasing the weight of each factor needs to be performed to tune the system. This can be ad-hoc and tedious; however, empirically, we could conclude what kinds of factors are used when creating the graph affect the recommendation performance significantly more important than what weights are given to factors. Regarding the content factor, you may repeat the similar process, however, in this case, what factors are useful to identify similar items should be speculated. When context and contextual factors are both available, using them with the same proportion can be suggested as a initial setup for hybrid recommendation. 
It is a challenging problem to find the optimal settings, and it should be acknowledged that this guideline does not guarantee the optimal performance of our method; however, while conducting experiments, we could find settings for our proposed method to outperform several existing state-of-the art recommender algorithms by empirically selecting factors and their weights for two different applications.

\section{Conclusions and Future Work}

Majority of existing approaches consider recommendation as an 'optimization' problem with specific objectives functions for optimizing accuracy, novelty, diversity, serendipity, and etc. Generally, recommendation engine optimized for specific criteria are hard-wired into the systems and, therefore, provide only a predefined and fixed set of recommendations. In this paper, we investigated how we could enable flexible customization of recommendation using graphs. To this end, we presented a novel view of seeing recommendation problem as graph construction and node ranking problems. Accordingly, as a baseline approach, we proposed a naïve graph-based recommendation method that operates with a log-metadata graph as a baseline method, which is a heterogeneous graph constructed from user log and content metadata tables in relational databases. Although its simplicity, the proposed method provides competitive recommendation performance with the state-of-the art recommendation methods by taking advantages of heterogeneous information and their relationships.

The remaining problem to be solved is that the scale of log-metadata graph is usually very large so that it could cause the inefficiency in terms of processing time. To tackle the problem, we propose a method to construct a compact sized graph that can be utilized for recommendation without losing the recommendation performance. Recommendation results with various settings and semantics can be effectively and efficiently generated by using the proposed method. Our proposed recommendation methods provide a systematic customization to perform various recommendations such as collaborative filtering, context-aware collaborative filtering, and hybrid recommendation changing different factor weights without much engineering efforts can be performed. We believe that such flexibility, the capability of easy customization, is one of the most the critical features for the agile reflection to users' fast changing behaviors.

To validate of our presented methods, we tested the recommendation performances of several different setting with empirically selected weights. Note that finding the optimal set of factors and their weights was beyond the scope of this research; however, by empirically selecting factors and their weights, we could find settings for our proposed methods to outperform several existing state-of-the art recommender algorithms with two different real world data sets. At the same time, we confirmed that our methods could significantly reduce the size of graph by constructing Factor-Item bipartite graph in comparison with a log-metadata graph without sacrificing the recommendation performance.

Moreover, it is a significant advantage that our methods can leverage various existing tools. For example, largegraph processing tools such as Pregel (Malewicz et al., 2010) and GraphX (Xin et al., 2013) along with our proposed method to maximize the efficiency of constructing and processing large-scale graphs for recommender systems. Recent approaches to process graphs on a PC or a mobile device, such as Kyrola et al. (2012), Han et al. (2013), and Chen et al. (2014) create a great synergy with our presented methods to realize online graph-based recommendations on a device with limited resources, where our methods utilize relatively smaller-sized graphs.

There are more interesting research challenges that can be considered as future work to resolve limitations of the proposed methods as follows:

Finding Optimal Weights using Machine Learning Techniques: In this paper, we did not consider the recommendation problem as optimization problem, as a result, we could give much freedom and flexibility to service providers; however, we believe that we could incorporate machine learning techniques into our proposed methods that operate as suggestions for service providers. We need to investigate how machine learning techniques can be exploited to optimize factors and weights with respect to various criteria not only accuracy, but also novelty, serendipity, diversity, etc. It is challenging to find a general way to guarantee the optimal settings for various criteria. However, we think greedy exploration with automated sampling can be applied as a baseline approach. For example, all possible factors are used with the same given weights, then iteratively update each weight after testing the performance regarding the selected performance measure for randomly sampled query nodes.

Developing Interactive Experiment Tools for Exploring Effective Settings: By taking advantage of flexibility, service providers can adjust and customize many settings for recommendation in our methods. However, currently, 
there is no systematic support for measuring performance with respect to various criteria while changing settings (e.g., factors, weights). It will be meaningful to provide a generalized system that allows service providers to perform experiments to investigate how the changes in query node types, factors, and weights affect various recommendation metrics. We expect that such system will be very helpful to service providers while exploring the settings which reflects rapidly changing users' preferences.

Constructing Large-scale Graph in a Scalable Way \& Incrementally Updating Graphs: When input data is extremely large and often updated, so, rebuilding whole graph can be time-consuming. Thus, in future work, we plan resolve the problem in two different ways. The first approach is to develop a scalable graph construction system which can leverage the recent parallel programming model, Map-Reduce (Dean and Ghemawat, 2008). The second one is to build a incremental graph update method so that it can synchronize input data and constructed graph. The first one is useful to speed up the initial graph construction that is necessary at least once for each input data, and the second one is to avoid rebuilding the whole graphs when there is major changes in the settings.

Developing Intuitive and User-friendly Interface: Our goal is to build a unified graph-based recommender system that allows users to flexibly perform various recommendation requests by easily changing various queries and parameters. To realize such system, we need to design an intuitive and user-friendly interface. We expect this will give great opportunities to content providers and consumers in that they can test various recommendation requests to empirically find the best recommendation results.

\section{Acknowledgement}

This manuscript has been authored by UT-Battelle, LLC, under contract DE-AC05-00OR22725 with the U.S. Department of Energy. The United States Government retains and the publisher, by accepting the article for publication, acknowledges that the United States Government retains a non-exclusive, paid-up, irrevocable, world-wide license to publish or reproduce the published form of this manuscript, or allow others to do so, for United States Government purposes.

This material is based upon work supported by the National Science Foundation Graduate Research Fellowship Program under Grant No. DGE-1148903.

This work was supported by the National Research Foundation of Korea(NRF) grant funded by the Korea Government(MSIP) (No. 20110030812).

\section{References}

Aditya, B., Bhalotia, G., Chakrabarti, S., Hulgeri, A., Nakhe, C., Parag, P., and Sudarshan, S. (2002). Banks: Browsing and keyword searching in relational databases. In Proceedings of the 28th International Conference on Very Large Data Bases, pages 1083-1086. VLDB Endowment.

Adomavicius, G., Sankaranarayanan, R., Sen, S., and Tuzhilin, A. (2005). Incorporating contextual information in recommender systems using a multidimensional approach. ACM Transactions on Information Systems, 23(1):103145.

Adomavicius, G., Tuzhilin, A., and Zheng, R. (2011). REQUEST: A query language for customizing recommendations. Information Systems Research, 22(1):99-117.

Balmin, A., Hristidis, V., and Papakonstantinou, Y. (2004). Objectrank: Authority-based keyword search in databases. In Proceedings of the 30th International Conference on Very Large Data Bases, pages 564-575. VLDB Endowment.

Baltrunas, L. and Ricci, F. (2014). Experimental evaluation of context-dependent collaborative filtering using item splitting. User Modeling and User-Adapted Interaction, 24(1-2):7-34.

Chen, Y., Lin, Z., Pienta, R., Kahng, M., and Chau, D. H. (2014). Towards scalable graph computation on mobile devices. In Proceedings of the 2nd Workshop on Scalable Machine Learning: Theory and Applications. 
Cheng, H., Tan, P., Sticklen, J., and Punch, W. (2007). Recommendation via query centered random walk on k-partite graph. In Proceedings of the 7th IEEE International Conference on Data Mining, pages 457-462. IEEE.

Codina, V., Ricci, F., and Ceccaroni, L. (2013). Semantically-enhanced pre-filtering for context-aware recommender systems. In Proceedings of the 3rd Workshop on Context-awareness in Retrieval and Recommendation, pages 15-18. ACM.

Cremonesi, P., Koren, Y., and Turrin, R. (2010). Performance of recommender algorithms on top-n recommendation tasks. In Proceedings of the 4th ACM Conference on Recommender Systems, pages 39-46. ACM.

Davidson, J., Liebald, B., Liu, J., Nandy, P., Van Vleet, T., Gargi, U., Gupta, S., He, Y., Lambert, M., Livingston, B., et al. (2010). The youtube video recommendation system. In Proceedings of the 4th ACM Conference on Recommender Systems, pages 293-296. ACM.

Dean, J. and Ghemawat, S. (2008). Mapreduce: simplified data processing on large clusters. Communications of the ACM, 51(1):107-113.

Demovic, L., Fritscher, E., Kriz, J., Kuzmik, O., Proksa, O., Vandlikova, D., Zelenik, D., and Bielikova, M. (2013). Movie recommendation based on graph traversal algorithms. In Database and Expert Systems Applications (DEXA), 2013 24th International Workshop on, pages 152-156. IEEE.

Floyd, R. (1962). Algorithm 97: shortest path. Communications of the ACM, 5(6):345.

Fouss, F., Pirotte, A., Renders, J., and Saerens, M. (2007). Random-walk computation of similarities between nodes of a graph with application to collaborative recommendation. IEEE Transactions on Knowledge and Data Engineering, 19(3):355-369.

Göbel, F. and Jagers, A. (1974). Random walks on graphs. Stochastic processes and their applications, 2(4):311-336.

Goldberg, A. and Tarjan, R. (1988). A new approach to the maximum-flow problem. Journal of the ACM, 35(4):921940.

Gori, M. and Pucci, A. (2007). Itemrank: a random-walk based scoring algorithm for recommender engines. In Proceedings of the 20th International Joint Conference on Artifical Intelligence, pages 2766-2771. Morgan Kaufmann Publishers Inc.

Hagberg, A., Swart, P., and S Chult, D. (2008). Exploring network structure, dynamics, and function using networkx. Technical report, Los Alamos National Laboratory (LANL).

Han, W.-S., Lee, S., Park, K., Lee, J.-H., Kim, M.-S., Kim, J., and Yu, H. (2013). Turbograph: a fast parallel graph engine handling billion-scale graphs in a single pc. In Proceedings of the 19th ACM SIGKDD international conference on Knowledge discovery and data mining, pages 77-85. ACM.

Haveliwala, T. (2003). Topic-sensitive pagerank: A context-sensitive ranking algorithm for web search. IEEE Transactions on Knowledge and Data Engineering, 15(4):784-796.

Herlocker, J., Konstan, J., Borchers, A., and Riedl, J. (1999). An algorithmic framework for performing collaborative filtering. In Proceedings of the 22nd International ACM SIGIR Conference on Research and Development in Information Retrieval, pages 230-237. ACM.

Huang, Z., Chung, W., Ong, T., and Chen, H. (2002). A graph-based recommender system for digital library. In Proceedings of the 2nd ACM/IEEE-CS Joint Conference on Digital Libraries, pages 65-73. ACM.

Jeh, G. and Widom, J. (2002). Simrank: a measure of structural-context similarity. In Proceedings of the 8th ACM SIGKDD International Conference on Knowledge Discovery and Data Mining, pages 538-543. ACM.

Kahng, M., Lee, S., and Lee, S.-g. (2011a). Ranking in context-aware recommender systems. In Proceedings of the 20th International Conference on World Wide Web, pages 65-66. ACM. 
Kahng, M., Lee, S., and Lee, S.-g. (2011b). Ranking objects by following paths in entity-relationship graphs. In Proceedings of the 4th workshop for Ph. D. students in information $\mathcal{F}$ knowledge management, pages 11-18. ACM.

Karatzoglou, A., Amatriain, X., Baltrunas, L., and Oliver, N. (2010). Multiverse recommendation: n-dimensional tensor factorization for context-aware collaborative filtering. In Proceedings of the fourth ACM Conference on Recommender Systems, pages 79-86. ACM.

Koren, Y. (2008). Factorization meets the neighborhood: a multifaceted collaborative filtering model. In Proceedings of the 14th ACM SIGKDD International Conference on Knowledge Discovery and Data Mining, pages 426-434. ACM.

Koutrika, G., Bercovitz, B., and Garcia-Molina, H. (2009). Flexrecs: expressing and combining flexible recommendations. In Proceedings of the 35th SIGMOD International Conference on Management of Data, pages 745-758. ACM.

Kyrola, A., Blelloch, G. E., and Guestrin, C. (2012). Graphchi: Large-scale graph computation on just a pc. In OSDI, volume 12, pages 31-46.

Lee, D., Park, S., Kahng, M., Lee, S., and Lee, S.-g. (2010). Exploiting contextual information from event logs for personalized recommendation. Computer and Information Science 2010, pages 121-139.

Lee, S., Park, S., Kahng, M., and Lee, S.-g. (2013). Pathrank: Ranking nodes on a heterogeneous graph for flexible hybrid recommender systems. Expert Systems with Applications, 40(2):684-697.

Lee, S., Song, S.-i., Kahng, M., Lee, D., and Lee, S.-g. (2011). Random walk based entity ranking on graph for multidimensional recommendation. In Proceedings of the fifth ACM conference on Recommender systems, pages 93-100. ACM.

Malewicz, G., Austern, M. H., Bik, A. J., Dehnert, J. C., Horn, I., Leiser, N., and Czajkowski, G. (2010). Pregel: a system for large-scale graph processing. In Proceedings of the 2010 ACM SIGMOD International Conference on Management of data, pages 135-146. ACM.

Page, L., Brin, S., Motwani, R., and Winograd, T. (1999). The pagerank citation ranking: Bringing order to the web.

Panniello, U., Tuzhilin, A., and Gorgoglione, M. (2014). Comparing context-aware recommender systems in terms of accuracy and diversity. User Modeling and User-Adapted Interaction, 24(1-2):35-65.

Pazzani, M. and Billsus, D. (2007). Content-based recommendation systems. The adaptive web, pages 325-341.

Rendle, S. (2010). Context-aware ranking with factorization models, volume 330. Springer-Verlag New York Inc.

Schölkopf, B. and Smola, A. (2002). Learning with kernels: Support vector machines, regularization, optimization, and beyond. The MIT Press.

Shi, C., Kong, X., Yu, P., Xie, S., and Wu, B. (2012). Relevance search in heterogeneous networks. In Proceedings of the 15th International Conference on Extending Database Technology, pages 180-191. ACM.

Sun, Y. and Han, J. (2012). Mining heterogeneous information networks: principles and methodologies. Synthesis Lectures on Data Mining and Knowledge Discovery, 3(2):1-159.

Sun, Y., Han, J., Yan, X., Yu, P., and Wu, T. (2011). Pathsim: Meta path-based top-k similarity search in heterogeneous information networks. In Proceedings of the 37th International Conference on Very Large Data Bases, volume 4. VLDB Endowment.

Whitman, B. and Lawrence, S. (2002). Inferring descriptions and similarity for music from community metadata. In Proceedings of the 2002 International Computer Music Conference, pages 591-598. Citeseer. 
Xiang, L., Yuan, Q., Zhao, S., Chen, L., Zhang, X., Yang, Q., and Sun, J. (2010). Temporal recommendation on graphs via long-and short-term preference fusion. In Proceedings of the 16th ACM SIGKDD International Conference on Knowledge Discovery and Data Mining, pages 723-732. ACM.

Xin, R. S., Gonzalez, J. E., Franklin, M. J., and Stoica, I. (2013). Graphx: A resilient distributed graph system on spark. In First International Workshop on Graph Data Management Experiences and Systems, page 2. ACM.

Yao, W., He, J., Huang, G., Cao, J., and Zhang, Y. (2014). A graph-based model for context-aware recommendation using implicit feedback data. World Wide Web, pages 1-21. 